\title{
Hardware-Software Design of a Smart Sensor for Fully-Electronic DNA Hybridization Detection
}

\author{
Claudio Stagni, Carlotta Guiducci, Massimo Lanzoni, Luca Benini, Bruno Riccò \\ DEIS-University of Bologna cstagni@deis.unibo.it
}

\begin{abstract}
This paper describes the design of a smart sensorfor label-free detection of DNA hybridization. The sensor is based on a direct electrical transduction principle: it measures impedance variation at the interface between a biofunctionalized electrode and a solution containing the anal-vte. The smart sensor includes a complete signal conditioning and processing subsystem based on an embedded $\mu$-controller. We outline the sensor architecture, and we describe in details board-level integration as well as hardware and software implementation and design choices. The accuracy of our embedded solution has been evaluated by comparing it with a high-cost laboratory setup. Moreover; we provide measurements of real sensing structures which demonstrate in field thefunctionality of our system.
\end{abstract}

\section{Introduction}

Smart sensors, which integrate transducers, signal conditioning and processing electronics in a single device, have played an important role in changing our society and lifestyle. These devices help people in collecting critical information on their environment and they often are embedded in complex control systems that automatically adapt environmental characteristics (e.g., temperature, luminosity) to the user's preferences. The last few years have witnessed an explosive growth of embedded applications for smart sensors. For example virtually every new car contains numerous smart sensors for engine control, safety and travel comfort [19]. The main motivation for the widespread diffusion of these devices is their low (and decreasing) cost, coupled with high flexibility and ease of deployment.

The implementation of a smart sensor is the end point of an evolutionary path whose key phases can be summarized as follows: (phase-])discovery of a sensing and transduction principle; (phase-2) quantitative analysis and modeling, (phase-3)development of high-cost sensors for laboratory applications, (phase-4)miniaturization and board-level integration based on commodity electronic components, (phase-5) single-chip integration. Deployment of smart sen- sors in embedded systems is possible starting from the fourth phase. In many cases single-chip integration is never achieved because of its high NRE cost and it many technology challenges.

In this paper we focus on bio-sensors and, more specifically, on genetic sensors, which can detect the key biomolecular interactions at the basis of gene expression processes. In this area, a new and important line of sensors development is based on the direct electrical detection of the main bio-molecular interaction in genetics, namely $D N A$ hybridization. Highly-parallel DNA hybridization detection systems (know as DNA-microarrays $[12,6]$ ) are widespread in laboratories, and they have rapidly become one of the main tools used by biologists for analyzing the genetic origins of biological processes. Microarrays are based on an optical sensing and transduction principle, which implies extra steps in processing input samples (a process known as labeling) as well as expensive optical transducers and postprocessing equipment.

For this reason, the leading commercial bio-sensors for DNA hybridization are "phase three" products: they are designed for laboratory usage, hence they are expensive, hard to use and not accessible to the general public or event to physicians for point-of-care usage. Several research efforts are under way, aiming at the development of new DNA hybridization detection systems with the potential for becoming phase-4 (andphase-5) smart sensors. These devices are generally characterized by label-free operation as well as direct electrical detection of hybridization. Even though several label-free direct electrical DNA hybridization sensors have been proposed in the literature $[13,18,16]$, none has been yet developed beyond phase- 1 or phase- 2 .

The main contribution of this paper is the first selfcontained design of a capacitive label-free DNA sensor based on COTS components (phase-4 sensor). Our implementation integrates the sensor within $\mu$-controllerbasedboard, which enables customization and self-tuning by exploiting the flexibility of programmable components. The accuracy of the phase- 4 implementation has been assessed by comparing it with a laboratory setup which interfaces the sensor with data acquisition and signal gen- 
eration instruments and manipulates incoming data with a PC equipped with standard test, measure and control software.

The paper is organized as follows: in the next section we provide basic background information on smart sensors and on DNA sensors. Section 4 describes the transduction principle. Section 5 describes the hardware and software design of our smart sensor. Section 6 details experimental results and provide a comparison with a high-cost laboratory setup. Conclusions are drawn in Section 7.

\section{Background}

In the present landscape of commercially available sensing devices, a key role is played by sensors fabricated using processes derived from the microelectronic industry. Among the main reasons of the success of these devices is that they are integrated in a limited space and at low cost with other (traditional) electronic parts dedicated to signal processing, data collection, interfacing and, possibly, wireless remote connection. All these functions, if not performed by the sensor device itself, result quite time and resource consuming and not so accurate as if performed insitu. Biosensors, i.e., sensors incorporating biological elements, are finding use in increasingly broader ranges of applications, such as clinical diagnosis food production and analysis, and many others. Within the broad field of biosensors, DNA sensors aim at the detection of the highlyspecific binding reaction between two molecules of DNA (hybridization). A detailed treatment of the biochemical basis of hybridization is beyond the scope of this paper (the interested reader is referred to [17] for an excellent introductory treatment). For our purpose, it is sufficient to understand that DNA molecules can be described as linear sequences of four different basic components (called nucleotides). DNA can be found in two states: single-stranded (i.e., a single linear sequence) and double-stranded. During hybridization, two single-stranded molecules bind to become a double-stranded molecule. This chemical reaction takes place (with high probability) only if the nucleotide sequences of the two single-stranded molecules match on a nucleotide-by-nucleotide basis. DNA biosensors use custom-designed single-stranded DNA molecules as recognizing elements (probes) for sub-sequences in the DNA of test samples. If hybridization between the probe and the sample DNA can be reliably detected, then the $g e$ netic signuture of the organism can be determined by virtue of the one-to-one affinity relationship.

\section{Previous Work}

Micro-arrays based on optical detection of DNAhybridization represent the state of the art $[10,9]$ and are now are capable of testing hundreds of thousands different probes in parallel (Fig. 1). Each site of the array hosts probes with a unique sequence. Sample sequences which need to be identified are labelled with chromophores molecules and spread on the array surface. After specific recognition between the sample and its complementary probe the device is observed optically to localize the chromophores, revealing the unknown sample sequence. This technology presents two main limitations: the cost of the instrument which has to detect and transduce the optical signal and, as already mentioned, the necessity of a labelling step which implies additional reagents and can lead to sample pollution. One straightforward di-

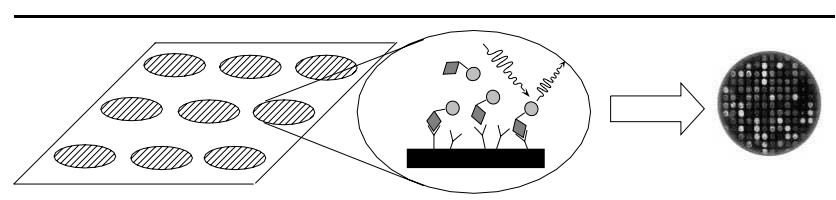

Figure 1. Microarray detection technique.

rection of evolution, which addresses the first limitation of DNA microarray, is fully electrical detection. It still exploits labels (enzymes) bound on the target DNA, which generate red-ox currents upon hybridization, when the solution containing the sample is polarized with a potential difference [8]. The obvious advantage of this approach is avoiding external complex and expensive optical instruments without decreasing performance, in particular sensibility, of the sensors with respect to optical detection.

To further advance the state of the art, researchers are developing label-free techniques to avoid the modification of DNA targets, further reducing costs and time [15, 18, 4, 9, $16,2,14]$. Among these attempts, a fully-electrical technique based on the measurement of the changes of electrical properties (impedance) of the substrate/electrolyte interface has been implemented starting from standard electrochemical interface analysis methodologies as chronoamperometry [2], impedance spectroscopy [14] or exploiting field effect devices [16]. The most relevant disadvantage of these techniques is the use of a three-electrode set-up that increases the complexity of the system. Commercial implementations of sensors based on impedance label-free detection have still not been presented.

\section{Sensing Principle}

Our system is based on an electrochemical approach which exploits a label-free detection technique based on capacitance measurements of bio-modified electrode/solution interfaces [7]. It requires the immobilization of single- 
stranded probe DNA molecules of known sequence on a conducting electrode surface (electrode functionalization). Functionalized electrodes are then immersed in a physiologic solution. When target DNA molecules (coming from a biological sample) are introduced in the solution they bind to the probes only if their sequences match. The binding of sample molecules on a functionalized electrode changes the impedance of the electrode-solution interface. The measurement set-up is based on an electrochemical cell which consists of two electrodes immersed in an electrolyte solution. The interface can be modeled with capacitance and resistance as shown in Fig. 2.

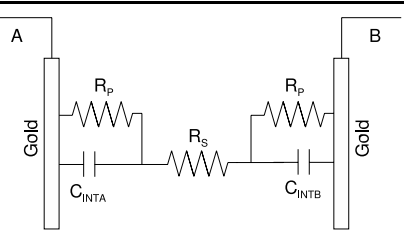

Figure 2. Electrode/solution interface model

The DNA interface modification introduces insulating layers between electrode and solution and the value of the capacitive component is strongly affected by their chemicalphysical characteristics. When DNA hybridization occurs, target molecules are blocked near the surface and change the structure of the interface, in particular they increase the distance between electrode and solution, thereby modifying the impedance of the interface. The charge-based capacitance measurement (CBCM) technique [5] can measure the variation of the capacitive component of the electrodesolution interface impedance. The principle of this technique is to charge and discharge, at an appropriate frequency, the impedance under test and to measure its equivalent capacitance from the average current in a half-period (Fig. 3). In fact, ideally, average current is proportional to capacitance, to frequency and to the step voltage applied to the capacitance itself, as shown in the following equation:

$$
I_{a v g}=\frac{\Delta Q}{T / 2}=\frac{C \Delta V}{=T / 2}=2 C \Delta V f
$$

Where $\Delta \mathrm{V}$ is known. To extract capacitance, one can measure $I,{ }_{t}$, ? for different frequencies and calculate the slope of the interpolated curve $I_{\text {avg }}$ versus $f$. This method has been widely employed for the measurement of on-chip parasitic capacitances [3] reaching very high accuracy limits. Alternatively, the current signal can be converted to a digital voltage signal and processed by a PC to perform averaging and capacitance extraction (Fig. 3).

The main advantage of CBCM technique compared to standard complex impedance meters based on analog sinusoidal signals is that it lends itself to a simple implementa-

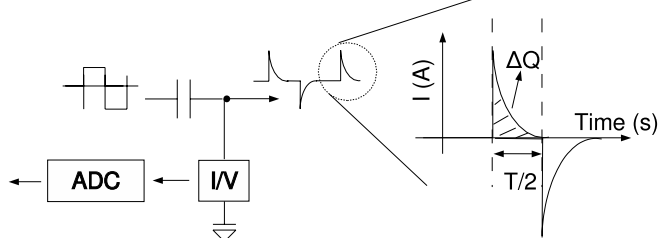

Figure 3. Principle of $\mathrm{CBCM}$ principle

tion using digital input signals. In fact, the analog part can be reduced to an I/V converter followed by an AID converter. A laboratory setup for performing CBCM requires a signal generator, to control the switches, and a current meter. The data coming from the current meter must then be processed by a PC to perform averaging and capacitance extraction.

\section{Hardware and Software Design}

The purpose of our design is to develop a low cost system to replace all the laboratory instruments that are connected to the CBCM cell, as well as the data-processing software running on the PC. The output of the system is in digital form, and can be either directly visualized on a LCD or forwarded to other subsystems through standard digital links (e.g. RS232, USB, etc.). Our implementation is based on $\mathrm{p}$-controller that processes directly the signals produced by the cell. The $\mathrm{p}$-controller also controls pulse generation (frequency and amplitude) by mean of a DAC converter, processes data sampled by the ADC converter and calculates $I_{\text {avg }}$ in charge and discharge cycles. In the next subsection we describe in detail both the hardware and software design of our smart sensor.

\subsection{Hardware}

As shown in Fig. 4 the system set-up is based on $\mu$ controller that controls one DAC device, to generate a square wave input signal to the cell, and an ADC device which samples and sends output cell current values (converted to voltages) to the $\mu$-controller. On the board there is also an OP-AMP with tunable gain to amplify output signal. At the end of the measurement, the capacitance value is available in digital form on a serial interface. The current/voltage conversion is performed with a precision resistor of appropriate value related to the capacitance range.

The p-controller is an ATMega 128 [1]: 8-bit, high performance, low power consumption and very low cost. It is the core of the system, since it controls time and synchronization among all components. The possibility to program this device is the most important point to guarantee flexibility and portability. 


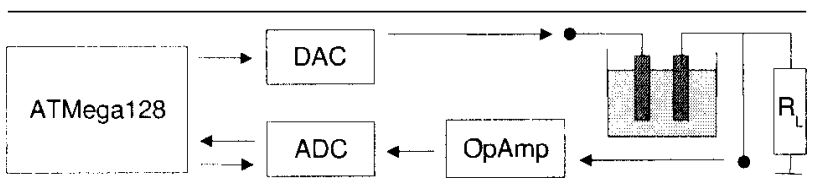

Figure 4. Block diagram

The DAC is a MAX536 [11] with four Out-channels and 16 bits program word The upper value $(2.048 \mathrm{~V})$ is generated by an ADR290 and the lower value $(-1.2 \mathrm{~V})$ by means of a zener diode LT1034. The control word is sent every time it is necessary to change the output signal, exployting an SPI protocol. The TL081 component, used to amplify the output signal, is a low noise OPAMP and it is connected with a variable feed-back resistance to regulate gain. The ADC is a MAX186[11]: a 12 bits and 8 channels converter. In case of both positive and negative values (as in our case), a 2's complement integer between $-V_{R E F A D C} / 2$ to $+V_{R E F A D C} / 2$ is generated.

\subsection{Software}

The programming languages used for ATMega 128 [1] are $\mathrm{C}$ and Assembler. First of all, the program identifies in a self-tuning step, the maximum frequency that allows correct measurement (see below), then it performs $I_{a v g}$ measurements at different frequencies and it finally calculates capacitance. The square wave is generated from the DAC which receives control words from ATMega 128, changes and holds its output value until the next command. The frequency is controlled by means of a counter in the ATMega 128, that generates an interrupt twice in a period.

Data acquisition starts just after the square wave edge. The ATMega128 sends ADC control bits, waits until the ADC has sampled and converted the data, The sampling time window should be wide enough to allow communication of 32 bit plus the time for sampling and conversion. If these operation are performed sequentially, the maximum achievable sample rate is $25 \mathrm{KHz}$. To increase performance, the ADC allows also a parallel Input/Output data transfer which increases sample rate to $40 \mathrm{KHz}$.

The CBCM technique assumes that the charge (or discharge) transient are completed (total charge is measured as the area under the current waveform when the transient has stabilized). In general, the capacitance value, hence the time constant, is not known a priori. To guarantee correct operation, a tuning step is required before starting the measurement: this step determines the maximum frequency to perform correct measurements.

In a laboratory setup, this tuning step is manually performed. To automate self-tuning, we implemented an iterative procedure that compares the last part of the transient obtained with progressively decreasing frequencies: if the dif- ference is below a small threshold defined a priori, the frequency is low enough to finish the discharge (or charge) transient and the current value reaches a constant value. The procedure starts from the maximum frequency, takes 25 samples before the following square-wave edge, calculates partial areas, and compare their areas: if the condition imposed by the threshold is verified for 10 consecutive transients, then the frequency can be used for the measurement. If this condition is not verified, the frequency is decreased and the procedure is repeated (Fig. 5).

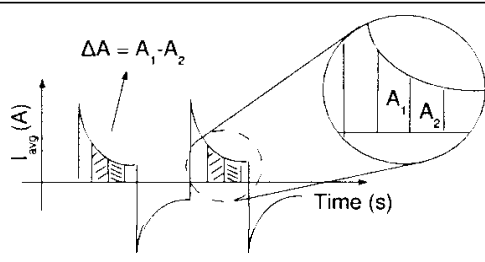

Figure 5. Tuning principle

When the system finds the maximum frequency, the test is stopped and a variable is set. Starting from this value, the function setRange generates a vector of frequencies which belong to this new range. The average time that is needed to perform this preliminary test is around $1 s$, that is compatible with biological systems.

After self-tuning, capacitance can be correctly measured. To do that, in principle it is necessary to calculate average currents for different frequencies and then calculate capacitance from equation (1). The system measures average voltage, which is proportional to current thanks to the resistor between the output cell and ground (Fig. 4). The following equation also considers the gain due to the OP-AMP $\left(A_{v}\right)$ and the current-to-voltage resistance $R_{L}$ :

$$
V_{a v g}=I_{a v g} R_{L} A_{v}=2 C R_{L} A_{v} \Delta V f=m f
$$

Therefore, assuming ideal capacitive behavior, it is possible to calculate $m$ as the slope of the linear interpolated curve by least squares fitting and to calculate the capacitance from the following equation:

$$
C=\frac{m}{R_{L} \cdot A_{v} \cdot 2 \cdot \Delta V}
$$

Capacitance is calculated sampling 10 half-period voltage transients. Each transient is sampled till $250 \mu \mathrm{s}$ before the end of the half-period to timely enable the $\mu$-controller interrupts, that are disabled during ADC sampling. In fact, an internal counter generates an interrupt twice in a period to warn the $\mu$-controller to change the DAC output voltage. Nevertheless, if the frequency has been defined according to the previous tuning process, the transient is expected to end at least $625 \mu \mathrm{s}$ before the end of the half-period. 


\section{Experimental Results}

The main electronic components used to build the system are off-the-shelf and inexpensive. Therefore, the cost of the complete system-on-board can be estimated of about $150 €$ mainly determined by the $\mathrm{p}$-controller, $\mathrm{ADC}$ and DAC components. Instead, the overall cost for a Lab-equipment set-up can be extimated to be around ten thousand euros (in this case virtual instrumentation is considered). The power consumption has been evaluated in idle state and during measurement: its average value is $520 \mathrm{~mW}$ without important variations during measurement. The system has been tested on a wide range of discrete capacitances (from $10 p F$ to $4.7 \mu F$ ) (section 6.2), and it has also been employed to determine the equivalent capacitance of an electrochemical two-electrode cell. The tuning procedure has been tested with $2.2 \mu F$ and $4.7 \mu F$ discrete capacitances to verify the correct estimation of maximum frequency (section 6.1).

\subsection{Tuning}

The tuning procedure determines $f_{M A X}$ the maximum frequency which allows the RC circuit (load resistor-cell capacitance) to be completely charged and discharged. This is the highest fequency for which Equation 2 is verified. Hence, the linear relationship between $f$ and $V$ predicted by Equation 2 is observed for all frequencies lower than $f_{M A X}$. The value of $f_{M A X}$ can be clearly observed on average voltage-frequency plot. For frequencies higher than $f_{M A X}$, the linear relationship is lost, as shown in Fig.6. At the maximum frequency determined after self-tuning, the deviation from the linear behavior is always below $1 \%$, thereby confirming the robustness and accuracy of the procedure described in section 5.2.

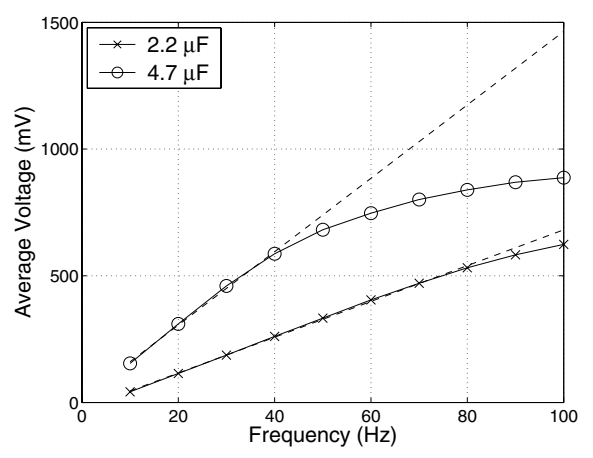

Figure 6. Deviation from linear behavior

\subsection{Performance comparison}

A Lab-equipment and the $\mu$-controller set-up are compared for a capacitance range starting from $10 p F$ to $4.7 \mu F$. In particular, we focused on two capacitance ranges that are characteristic of macro $\left(1 \mathrm{~cm}^{2}\right)$ and micro $\left(10^{3} \mu \mathrm{m}^{2}\right)$ electrodes, respectively. The macro-electrodes range was chosen from $350 n F$ to $4.7 \mu F$ while the micro-electrodes range was chosen from $10 p F$ to $700 p F$, according to available data [2] which associates $20 \mu \mathrm{F}$ capacitance for $\mathrm{cm}^{2}$ to an electrode/solution interface. The current to voltage conversion resistor was $750 \Omega$ for larger capacitances and $2.3 \mathrm{M} \Omega$ for smaller ones, respectively. Results related to four large capacitances are shown in Tab. 1, comparing the two measurement systems. Relative errors associated with capacitance measurements are below $3.5 \%$ for both set-ups.

\begin{tabular}{|c|c|c|c|c|}
\hline CAP $(\mu F)$ & \multicolumn{2}{|c|}{ Lub-equipment } & \multicolumn{2}{|c|}{-controller } \\
\hline & $C(\mu F)$ & Error $(\%)$ & $C(\mu F)$ & Error $(\%)$ \\
\hline 4.7 & 4.63 & 1.4 & 4.66 & 0.82 \\
\hline 2.2 & 2.18 & 0.94 & 2.13 & 2.87 \\
\hline 1 & 9.68 & 3.23 & 9.90 & 0.92 \\
\hline 0.35 & 0.341 & 257 & 0.356 & 18 \\
\hline
\end{tabular}

Table 1. Comparison between the two systems from $350 n F$ to $4.7 \mu F$ capacitance range

Lab-equipment and $\mu$-controller set-ups have been tested in the range of $10 p F$ to $700 p F$ capacitance to estimate the performance expected on micro-electrode cells $\left(1000 \mu \mathrm{m}^{2}\right)$. In both cases, the curve of experimental data versus nominal values has been plotted and the linear interpolation has been used as a calibration curve.

The calibration is necessary in particular for the Labequipment implementation because it presents a positive shift of about $50 \mathrm{pF}$ compared to the nominal value, instead, the $\mathrm{p}$-controller system presents a shift of only $2-3 p F$ (Fig. 7). The reduction of parasitics is a consequence of the more compact on-board implementation. Nevertheless, exploiting the calibration curves it is possible to eliminate the systematic error.

\begin{tabular}{|c|c|c|}
\hline \multicolumn{3}{|c|}{$150 p F$ caoacitance value } \\
\hline & Measurement $(p F)$ & Error (\%) \\
\hline Lub-equipment & 147.8 & 1.46 \\
\hline$u^{m}$ controller & 149.34 & 0.43 \\
\hline
\end{tabular}

Table 2. $150 \mathrm{pF}$ measurement test

Performing measurements of known capacitance in the micro-electrode range and exploiting calibration curves, it is possible to estimate the error with respect to the nominal 


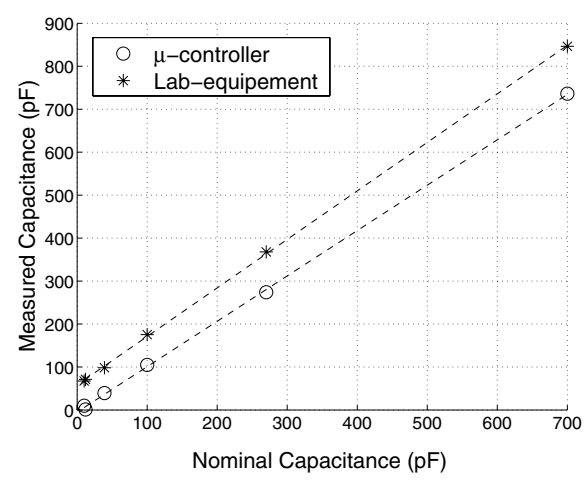

Figure 7. Calibration curves

value and compare the two systems. An example of measurement using $150 p F$ capacitance is shown in Tab. 2. Results show tha the $\mathrm{p}$--controller board exhibits smaller errors on capacitance measurement even in this range.

\subsection{Electrochemical two-electrode cell}

The $\mu$-controller set-up has been employed to measure the equivalent capacitance of an electrochemical sensing system. The system-on-board has been connected to a cell of two $0.8 \mathrm{~cm}^{2}$ gold electrodes immersed in an electrolyte solution. Three different cells have been tested, bare gold electrodes, one bare gold and a chemo-modified gold $\left(\left(\mathrm{SH}\left(\mathrm{CH}_{2}\right)_{11} \mathrm{COOH}\right)\right)$, one bare gold and a bio-modified gold (thiol-ended single-stranded 30-mer oligonucleotides). As the equivalent capacitance is mainly determined by the gold/solution interface capacitances, bare gold capacitance can be calculated from the first cell measurement as $19.04 \mu \mathrm{F} / \mathrm{cm}^{2}$. Chemo and bio-modification of the gold surface leads to an interface capacitance decrease. The entity of the variation is related to the biochemical nature of the molecular layer formed on the surface and to its conformation. In fact, a lower capacitance value has been measured for the cells that hosted chemo- and bio-modified electrodes. In particular, chemo-modified gold exhibits a capacitance of $1.42 \mu \mathrm{F} / \mathrm{cm}^{2}$ while bio-modified exhibits a capacitance of $4.37 \mu \mathrm{F} / \mathrm{cm}^{2}$. In agreement with literature values [14], chemo-modification leads to a higher capacitance decrease.

\section{Conclusions}

In this work we have developed and tested an embedded system-on-board for DNA detection. The main advantages of this DNA sensor implementation compared to commercially available systems are to avoid the use of high-cost detectors and the pre-treatment of the sample to introduce marker molecules. Moreover, the system is stand-alone and low-cost. Self-tuning functionality, which has been imple- mented by means of a $\mathrm{p}$-controller feedback procedure has been evaluated. The system has also been tested and compared in terms of accuracy with a laboratory high-cost implementation of the same charge-based capacitive measurement technique, showing better performance. Moreover, a programmable $\mathrm{p}$-controller allows for high flexibility and multi-functionality of the system. In particular, the system could host and manage additional components, such as a wireless interface or other sensors

\section{References}

[1] Atmel. www.atmel.com.

[2] C. Berggren. A feasibility study of a capacitive biosensor for direct detection of DNA hybridization. Electroanalysis. 3(11):156-160, 1999.

[3] A. Bogliolo. Charge-based on-chip measurement technique for the selective extraction of cross-coupling capacitances. Signal Propagation on Interconnects, 2002.

[4] F. Caruso. Quartz crystal microbalance study of DNA immobilization and hybridization for nucleic acid sensor developement. Analytical Chemistry, 69(11):2043-2049, 1997.

[5] J. Chen. An on-chip, attofarad interconnect charge-based capacitance measurement (CBCM) technique. International Electron Devices Meeting, 1996.

[6] D. Gershon. Microarray technology. An array of opportunities. Nature, 416(6883):885-891, 2002.

[7] C. Guiducci. DNA detection by integrable electronics. Biosensors and Bioelectronics, 19(8):78 1-787, 2004.

[8] F. Hofmann. Fully electronic DNA detection on a CMOS chip: device and process issues. International Electron Devices Meeting, 2002.

[9] Z. Junhui. DNA based biosensors. Biotechnology Advances, 15(1):43-58, 1997.

[10] G. C. Kennedy. Large-scale genotyping of complex DNA. Nature Biotechnology, 21(10):1233-1237, 2003.

[11] MAXIM. www.maxim-ic.com.

[12] M. C. Pirrung. How to make a DNA chip. Angewandte Chemie International Edition, 41(8): 1276-1289, 2002.

[13] R. L. Rich. Advances in surface plasmon resonance biosensor analysis. Current Opinion in Biotechnology. 11(1):54$61,2000$.

[14] M. Riepl. Optimization of capacitive affinity sensors: drift suppression and signal amplification. Analytica Chimica Acta, 392(1):77-84, 1999.

[15] M. Su. Microcantilever resonance-based DNA detection with nanoparticle probes. Applied Physics Letters, 82(20):3562-3564, 2003.

[16] F. Uslu. Labelfree fully electronic nucleic acid detection system based on a fi eld-effect transistor device. Biosensors and Bioelectronics, 19(12):1723-1731, 2004.

[17] J. D. Watson. Molecular biology of the gene. Fifth Edition. Benjamin-Cummings Publishing Company, 2003.

[18] I. Willner. Amplified detection of single-base mismatches in DNA using microgravimetric quartz-crystal-microbalance transduction. Talanta, 56(5):847-856, 2002.

[19] J. Xu. Vision guided automatic parking for smart car. IEEE Intelligent Vehicles Symposium, 2000. 